Document downloaded from:

http://hdl.handle.net/10251/92202

This paper must be cited as:

Ortiz Serna, MP.; Carsí Rosique, M.; Redondo Foj, MB.; Sanchis Sánchez, MJ.; Culebras, M.; Gomez, CM.; Cantarero, A. (2015). Thermal and dielectric properties of polycarbonatediol polyurethane. Journal of Applied Polymer Science. 132(22):42007-142007-8. doi:10.1002/app.42007

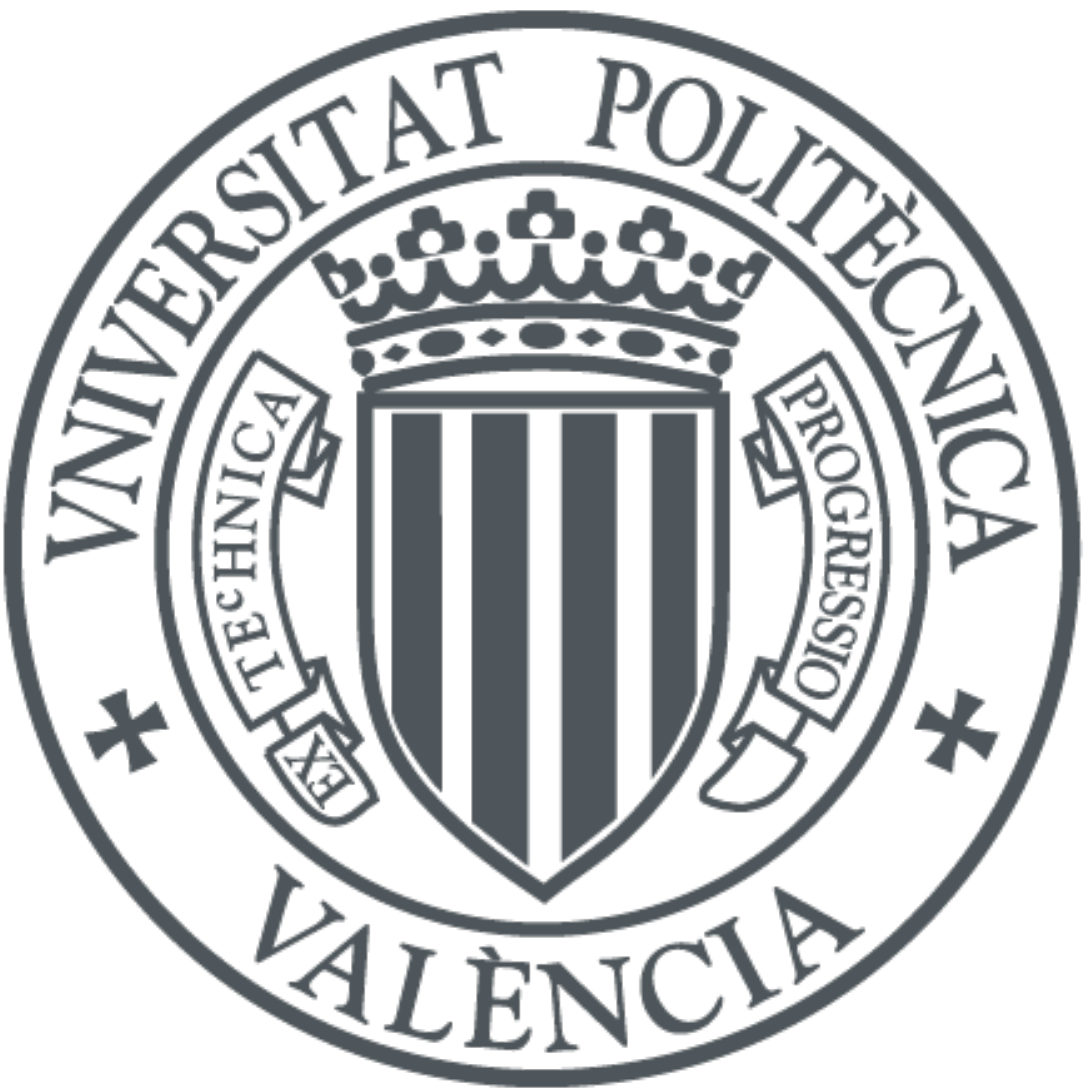

The final publication is available at

http://doi.org/10.1002/app.42007

Copyright John Wiley \& Sons

Additional Information 


\title{
Thermal and Dielectric Properties of Polycarbonatediol Polyurethane
}

\author{
P. Ortiz-Serna ${ }^{1}$, M. Carsí ${ }^{1}$, B. Redondo-Foj ${ }^{1}$, M. J. Sanchis*1, M. Culebras ${ }^{2}$, C.M. Gómez ${ }^{2}$ and A. Cantarero ${ }^{2}$ \\ ${ }^{1}$ Departamento de Termodinámica Aplicada, E.T.S.I.I., Instituto de Tecnología Eléctrica Universitat \\ Politècnica de Valencia, Camino de Vera s/n, 46022 Valencia, Spain \\ ${ }^{2}$ Materials Science Institute, University of Valencia, P.O. Box 22085, 46071 Valencia, Spain \\ Correspondence to: M.J. Sanchis (E-mail: jsanchis@ter.upv.es)
}

\begin{abstract}
The dielectric relaxation behaviour of a segmented polyurethane has been studied using Broad-Band Dielectric Spectroscopy in the frequency domain, $10^{-2}-10^{9} \mathrm{~Hz}$, and in the temperature range of -120 to $140 \stackrel{\circ}{ }$. The spectra show three secondary processes $(\delta, \gamma$ and $\beta)$ followed by the $\alpha$ relaxation and conductive processes. The Havriliak-Negami (HN) phenomenological equation was used in order to characterize all the processes. The $\delta, \gamma$ and $\beta$ relaxations are probably associated with (i) local motions of the main chain (ii) motions of the carbonate group in the soft phase and (iii) reorientational motions of the water molecules. The microphase-separated morphology associated with soft and hard domains is reflected in the dielectric spectra, at high temperatures, by the presence of the Maxwell-Wagner-Sillars (MWS) interfacial polarization process.
\end{abstract}

KEYWORDS Polyurethane; microphase separation; dielectric spectroscopy; thermal analysis; relaxation process; conductivity processes

\section{INTRODUCTION}

Polyurethanes are one of the most versatile materials since they can behave as elastomers, thermoplastics or thermoset polymers depending on the different reactants and synthesis conditions selected. They are extensively used as foams, elastomers, coatings, sealants and adhesives in different applications. ${ }^{1-5}$ Segmented polyurethane elastomers (TPU) are linear block copolymers formed by thermodynamically incompatible segments named as soft and hard domains. The soft segment is a polyol of molecular mass between 500 and $3000 \mathrm{~g} \mathrm{~mol}^{-1}$ that provides high flexibility at room temperature. The hard segment is formed by a 
diisocyanate and a low-molecular-weight diol which provides physical crosslink sites through strong intermolecular hydrogen bonding. ${ }^{6-7}$ The morphological, physical, chemical and mechanical properties of TPUs range in a wide spectrum depending on the hard segment formulation and content, molecular structure of soft segment and manufacturing conditions. Polyether and polyester macrodiols are commonly used in a wide range of commercial applications due to their affordable price. In contrast, polycarbonatediol (PCD) derived TPUs depict improved water and organic solvent resistance, high mechanical strength and high heatresistance, at the expense of an increase in the price.$^{8-10}$ Due to their inherent properties they are used for specific applications as artificial and synthetic leathers, thermoplastics, elastomers, paints and adhesives, where high reliability is required. The wide range of properties attained is directly related to the chemical composition reflected by the different hard-hard and hard-soft segments interactions. Demand for PCD is growing in the World as the polyurethane market increasingly shifts toward higher-performance materials. In this respect, we have being analysing in the last years the improved efficiency of PCD based TPUs., ${ }^{71-12}$

The development of energy devices for different applications requires the understanding of the electrical behaviour of certain materials not yet investigated in depth. Novel materials with different phase-separated morphology have been prepared and the influence of morphologies on the molecular dynamics has been analysed. ${ }^{13-19}$ The dielectric behaviour can be directly related to phase-separated morphologies and is a suitable tool to understand the mechanisms underlying the physical properties. 
The objective of the present study is to analyse the effect of the hard/soft domains in the molecular dynamics of polycarbonatediol polyurethane. In order to analyse the molecular dynamics of this material, we have employed the dielectric spectroscopy because it is a useful technique that allows the study of dynamics over a very broad range of frequencies, both in the glassy and rubbery states.

\section{EXPERIMENTAL}

\section{Materials}

Polyurethane solutions were obtained by a standard polymerization method based on the twostep process in dimethylacetamide. ${ }^{7,20}$ 4,4'-diphenylmethane diisocyanate (MDI), 1,4butanediol (BD), and dimethyl acetamide (DMAC), as solvent, were purchased from Aldrich (Barcelona, Spain). The macrodiol Polyhexamethylene-pentamethylene carbonatediol (PH100) of molar mass $1000 \mathrm{~g} \cdot \mathrm{mol}^{-1}$ and glass transition temperature of $-51.32{ }^{\circ} \mathrm{C}$, was kindly supplied by UBE Chem Eur. (Castellón, Spain). All materials were kept in a dry box to avoid humidity. The molar ratio used for this study was $\mathrm{PH} 100: \mathrm{BD}: \mathrm{MDI}=1: 2: 3$ in mole ratio. In the first step, one equivalent of $\mathrm{PH} 100$ was inserted in the reactor, previously dried for $24 \mathrm{~h}$ at $100{ }^{\circ} \mathrm{C}$, with three equivalents of $4,4^{\prime}$-diphenylmethane diisocyanate. The reaction was carried out at $70^{\circ} \mathrm{C}$ for $2 \mathrm{~h}$ under argon atmosphere. The second step corresponds to the addition of the chain extender, butanediol. Two equivalents of butanediol were added to obtain a viscous polyurethane solution with approximately $25 \mathrm{wt} \%$ in solid content. The solution was stored for $24 \mathrm{~h}$ in order to remove the bubbles produced in the synthesis process. Then, it was deposited on glass slides, which were previously washed in an ultrasonic bath with water and acetone. The polyurethane coated glasses were kept at $70{ }^{\circ} \mathrm{C}$ during $12 \mathrm{~h}$. Films were prepared with dimensions of $(4 \times 2.7)$ 
$\mathrm{cm}^{2}$ and with a thickness that ranged between 200 and $250 \mu \mathrm{m}$. Scheme 1 shows the chemical structure of the segmented polyurethane (PUPH).

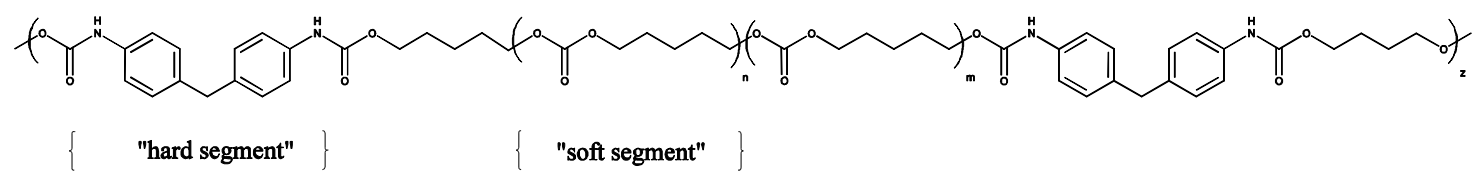

SCHEME 1 Chemical structure of the segmented polyurethane in soft and hard segments.

\section{Modulated Differential Scanning Calorimetry measurements}

Modulated Differential Scanning Calorimetry (MDSC) of polyurethane was carried out using a TA Instruments DSC Q-20 with a refrigerated cooling stage calibrated with indium and sapphire. The measurement was done in non-hermetic aluminium pans using an empty one as the reference cell from $-80{ }^{\circ} \mathrm{C}$ to $220^{\circ} \mathrm{C}$ at a heating rate of $3{ }^{\circ} \mathrm{C} \mathrm{min}-1$. The sample mass was $7.0 \pm$ $0.1 \mathrm{mg}$. The test was performed under a $50 \mathrm{ml} \cdot \mathrm{min}^{-1}$ flow of nitrogen to prevent oxidation. The modulation temperature amplitude was $\pm 1^{\circ} \mathrm{C}$ with a period of 60 seconds. The glass transition temperature $\left(T_{g}\right)$ was evaluated as the intersection of the base line of the glassy region with the tangent to the endotherm in the middle point.

\section{Dielectric measurements}

The experimental dielectric behaviour of PUPH was studied with a Novocontrol Broadband Dielectric Spectrometer (Hundsagen, Germany) consisting of an Alpha analyser to carry out measurements from $5 \cdot 10^{-2}$ to $3 \cdot 10^{6} \mathrm{~Hz}$ and an Agilent 4991A coaxial line reflectometer to carry out the measurement in the frequency range of $10^{6}$ to $10^{9} \mathrm{~Hz}$. In the latter case the complex permittivity was obtained by measuring the reflection coefficient at a particular reference plane. 
The measurements were carried out in inert $\mathrm{N}_{2}$ atmosphere between $-120^{\circ} \mathrm{C}$ and $140{ }^{\circ} \mathrm{C}$. The temperature was controlled by a nitrogen jet (QUATRO from Novocontrol), with a temperature uncertainty of $0.1{ }^{\circ} \mathrm{C}$ during every single sweep in frequency. Moulded disc shaped sample of about $0.1 \mu \mathrm{m}$ thicknesses and $20 \mathrm{~mm}$ diameter was used. The experimental uncertainty was better than $5 \%$ in all cases.

\section{RESULTS AND DISCUSSION}

\section{Modulated Differential Scanning Calorimetric Measurements}

MDSC was used in order to characterize the thermal behaviour of the polyurethane. This technique applies a sinusoidal temperature oscillation and separates the heat flow in two components, reversing and non-reversing heat flow. The reversing signal provides a high resolution to determine the glass transition temperature and the non-reversing signal is very useful to characterize the time-dependent behaviour, such as enthalpy relaxation and recrystallization. Figure 1 shows MDSC curves for PUPH. Two transitions are observed in the reversing signal. The first one is a glass transition temperature at $1.2^{\circ} \mathrm{C}$, with heat capacity $\Delta C_{p}=$ $0.149 \mathrm{~J} \mathrm{~g}^{-1}{ }^{\circ} \mathrm{C}^{-1}$, which is related to the amorphous part of the polyurethane. The $T_{g}$ increases 59.4 degrees with respect to that of the macrodiol $\left(\mathrm{PH} 100,-51.32{ }^{\circ} \mathrm{C}\right)$, indicating a great degree of miscibility of the soft segment with the hard segment. ${ }^{1,8,7,20}$ Miscibility between hard and soft segments results from their ability to establish hydrogen-bonding interactions. ${ }^{21}$ The second transition is an endotherm peak at $160{ }^{\circ} \mathrm{C}$, which is related to the melting of hard domains..$^{8,22-25}$ 
On the other hand, two transitions are observed in the non-reversing signal. The first one, located around $58{ }^{\circ} \mathrm{C}$, corresponds to the melting of hydrogen bonding domains between soft segment and $\mathrm{N}-\mathrm{H}$ groups of urethanes ${ }^{24,25}$. The second transition is the same endotherm peak observed in the reversing signal at $160{ }^{\circ} \mathrm{C}$, corresponding to the melting of hard domains. Overall, the transition at low temperature can be related to the soft segments reflecting the degree of mixing between soft and hard phases, while the transition at higher temperature is mainly related to the ordering of the hard segments.

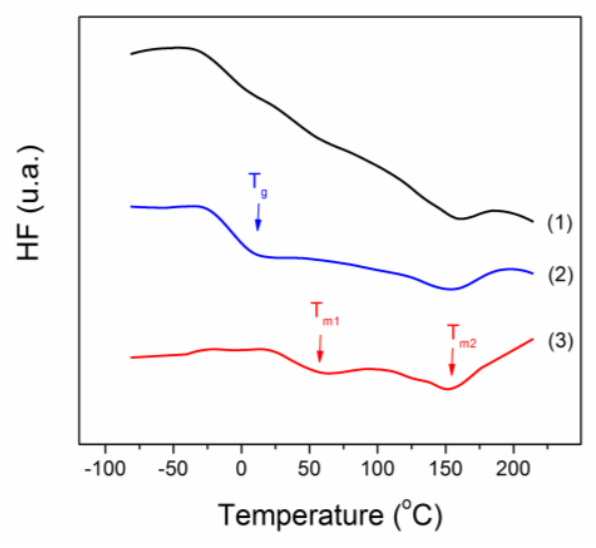

FIGURE 1 Modulated Differential Scanning Calorimetry curves of PUPH. (1) Total heat flow; (2) reversing component and (3) non-reversing component.

The thermal conductivity was determined by MDSC, according to the ASTM E1952-11 standard method. This is ideal for measuring thermal conductivity of polymeric materials with values between $0.1-1.0 \mathrm{~W} \mathrm{~m}^{-1} \mathrm{~K}^{-1}$. A value of $0.23 \pm 0.02 \mathrm{~W} \mathrm{~m}^{-1} \mathrm{~K}^{-1}$ was obtained for the thermal conductivity of PUPH in good agreement with previous results. ${ }^{26}$ The thermal diffusivity was calculated using the following equation (ASTM E1952-11):

$$
\alpha=\frac{\pi \cdot k \cdot d^{2} \cdot L}{4 \cdot C_{p} \cdot m}
$$


where $\kappa$ is the thermal conductivity; $d$ the specimen diameter; $L$ sample length; $C_{p}$ the heat capacity and $m$ the sample mass. A value of $0.17 \pm 0.02 \mathrm{~mm}^{2} \mathrm{~s}^{-1}$ was obtained for the thermal diffusivity of PUPH.

\section{Dielectric measurements}

Figure 2 shows three-dimensional (3D) representations of the real and imaginary components of the complex dielectric permittivity for the PUPH film, in the range of $5 \cdot 10^{-2}$ to $10^{9} \mathrm{~Hz}$ and temperature window of -120 to $140{ }^{\circ} \mathrm{C}$.

All the isochrones showing the variation of the dielectric permittivity $\varepsilon^{\prime}$, display the same pattern in the sense that they present two steps, a low temperature step associated with the glass rubber transition or $\alpha$ relaxation followed by a second step at higher temperature, associated with the Maxwell-Wagner-Sillars (MWS) relaxation. ${ }^{27-29}$ Finally, an important increase of the permittivity, related to the electrode polarization (EP) effects, it is observed. EP originates from the accumulation of charges at the electrode-polymer interface whereas the interfacial polarization or MWS process is due to the build-up of charges at the interfaces of the components of the heterogeneous systems. ${ }^{30}$ As discussed above, the PUPH is a heterogeneous system due to the existence of microphase separation associated with the soft and hard microdomains. The polarization of microdomains resulting from the separation of the hard and soft segments of PUPH chains can cause the accumulation of charges, a process that can be attributed to the different conductivity paths between the soft and hard microdomains. The 
evidence of the presence of these microdomains in the PUPH under study has been demonstrated by means of the WAXS measurement results. ${ }^{12}$
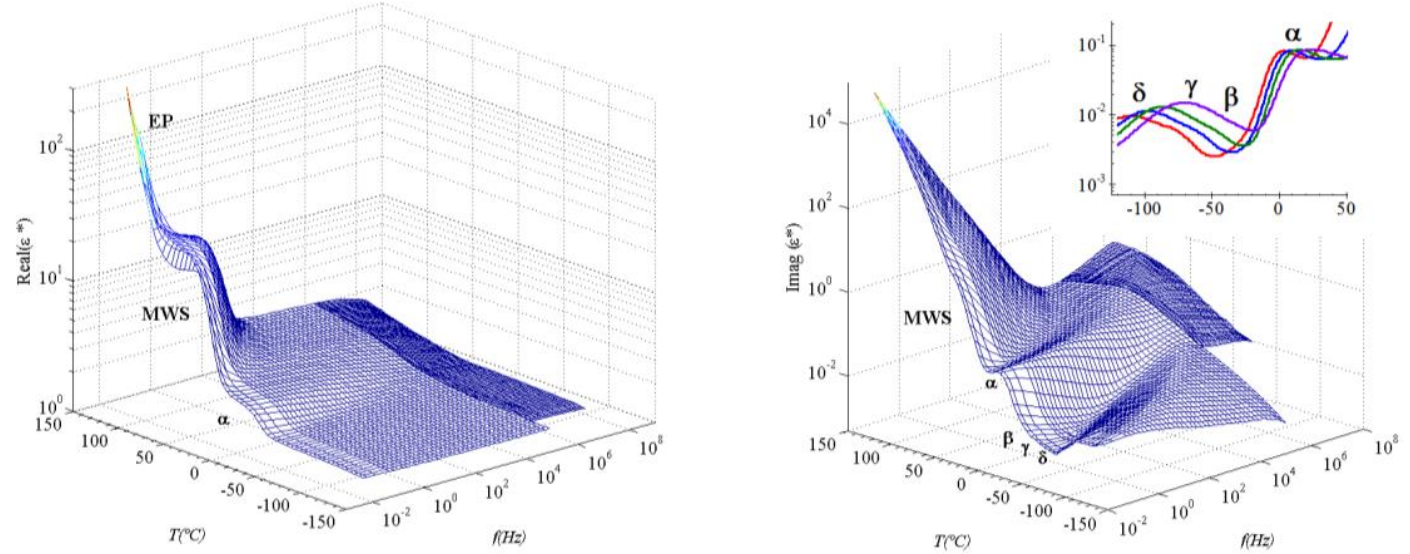

FIGURE 2 3D representation of the dielectric permittivity of PUPH in the temperature range of 120 to $140{ }^{\circ} \mathrm{C}$, step $5^{\circ} \mathrm{C}$. Inset: zoom of the low temperature region.

The isochrones corresponding to the dielectric loss $\varepsilon^{\prime \prime}$ clearly present two relaxational zones. In the low-temperature region, we can observe the presence of three secondary relaxations, labelled as $\delta, \gamma$ and $\beta$ processes, presumably associated with side chain motions (see inset in Fig. 2). In the high-temperature zone the spectrum present an ostensible $\alpha$ relaxation associated with the glass transition temperature followed by a rather sharp increase as temperature increases as a result of the strong contribution of the conductivity. The contribution of the polarization, produced at the electrode-polymer interface, to the dielectric loss scales with frequency dependence as $\omega^{-s}$, where usually $s$ is a parameter close to unity.

PUPH is a typical system in which charge contributions to the dielectric permittivity are important at low frequencies. Therefore, it is also convenient to analyse the results in terms of the complex dielectric modulus $M^{*}=1 / \varepsilon^{*}$, a variable very sensitive to charge transport. ${ }^{31}$ This 
formalism has several advantages: ${ }^{32}$ (i) it allows the determination of the $d c$ conductivity from the $M^{\prime \prime}$ spectra, (ii) electrode polarization effects usually do not mask the features of the spectra, and (iii) the results of the dielectric analysis are seemingly analogous to the mechanical modulus of solids. Figure 3 plots the mechanical $\left(E^{\prime \prime}\right)^{12}$ and dielectric loss modulus $\left(M^{\prime \prime}\right)$ as a function of temperature at $1 \mathrm{~Hz}$. Both spectra clearly present two relaxational zones. In the lowtemperature region, we can observe the presence of several secondary relaxations, presumably associated with local motions. In the high-temperature region, both spectra show an ostensible $\alpha$ relaxation. Moreover, only in the dielectric spectrum two processes associated with the conductivity phenomena (MWS and EP) are observed.

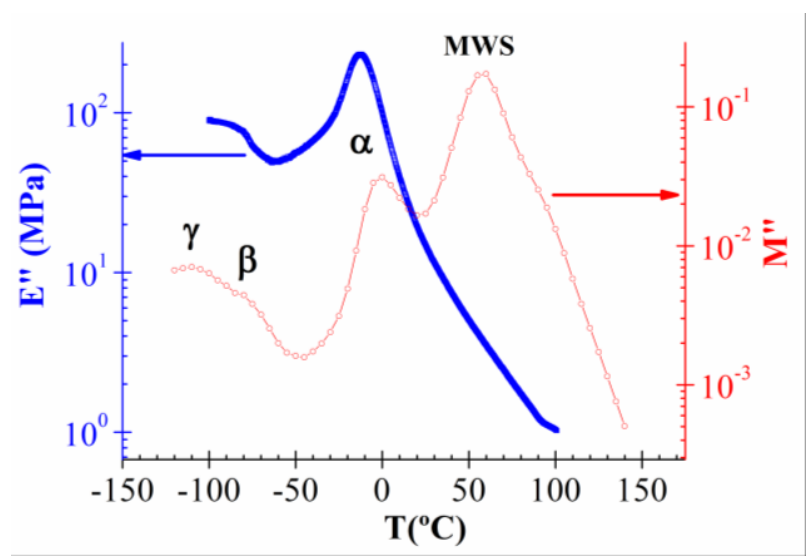

FIGURE 3 Temperature dependence of the dielectric loss modulus and loss mechanical modulus at $1 \mathrm{~Hz}$ for PUPH film.

The pertinent loss modulus in the frequency and temperature experimental range is shown in Figure 4. As we can see, the loss modulus exhibits two ostensible peaks corresponding to the $\alpha$ relaxation and the MWS process, in decreasing order of frequency. This last process is overlapped with the EP process in the low frequency region. 


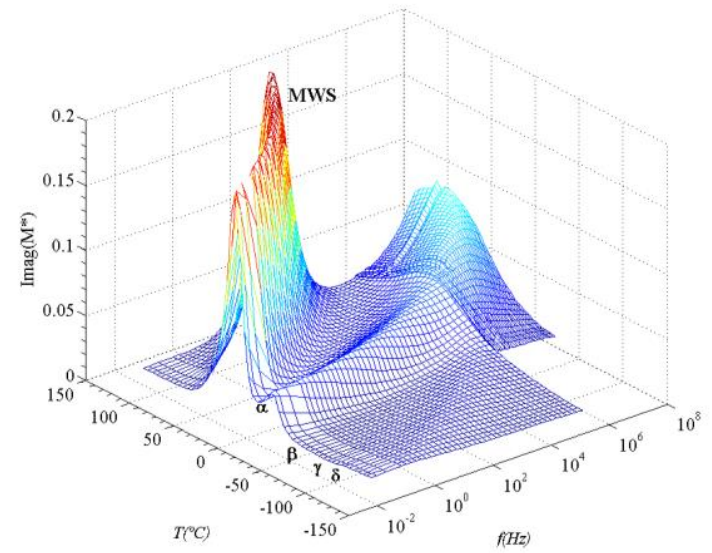

FIGURE 4 Values of $M^{\prime \prime}$ in the frequency domain for PUPH at several temperatures $(-120$ to $140{ }^{\circ} \mathrm{C}$, step $\left.5^{\circ} \mathrm{C}\right)$.

In the $\tan \delta$ representation (Fig. 5) it is observed a peak at lower frequencies than that of the glass-rubber relaxation. This peak is associated with the EP phenomenon that is dominant in the low frequency range.

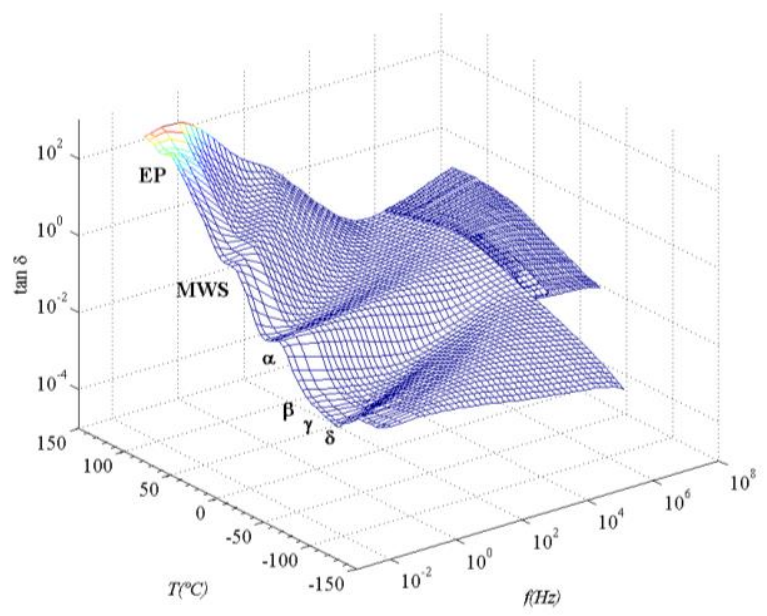

FIGURE 5 Loss tan $\delta$ in the frequency domain for PUPH at several temperatures $\left(-120\right.$ to $140{ }^{\circ} \mathrm{C}$, step $\left.5^{\circ} \mathrm{C}\right)$. 
The analysis of the dielectric spectrum was made by using the phenomenological equation of Havriliak-Negami (HN). ${ }^{33-34}$ This equation relates the complex permittivity $\left(\varepsilon^{*}\right)$ to the frequency by

$$
\varepsilon_{d i p(\omega)}^{*}=\varepsilon_{\infty}+\frac{\varepsilon_{0}-\varepsilon_{\infty}}{\left[1+\left(j \omega \tau_{H N}\right)^{\left.a_{H N}\right]^{b} H N}\right.}
$$

where the subscripts $\varepsilon_{0}$ and $\varepsilon_{\infty}$ are, respectively, the relaxed $(\omega \rightarrow 0)$ and unrelaxed $(\omega \rightarrow \infty)$ dielectric permittivities, and $\tau_{\mathrm{HN}}$ is the characteristic relaxation time associated with the relaxation. The shape parameters $a_{H N}$ and $b_{H N}$ fulfill the condition $0<a_{H N} b_{H N} \leq 1$, and for a Debye process $a_{H N}=b_{H N}=1$. These parameters are related, respectively, to the departure of the complex $\varepsilon^{\prime \prime} v s \varepsilon^{\prime}$ plot from a semi-circumference, at low frequencies, and to the skewness of the plot along a straight line, at high frequencies. ${ }^{35}$

The analysis of the dielectric spectrum was carried out in the two zones: $(i)$ high frequency zone where three secondary processes are intimately overlapped and (ii) low frequency zone where the $\alpha$ process is overlapped with a secondary process, at high frequency values, and with MWS and EP processes at low frequency values.

In the high frequency zone, an additive rule for the permittivity was assumed, ${ }^{36}$ i.e., the analysis of the dielectric spectra was carried out by means of the addition of two HN functions corresponding to $\beta$ and $\gamma$ processes (from -45 to $-70{ }^{\circ} \mathrm{C}$ ) and to $\delta$ and $\gamma$ processes (from -80 to $-110^{\circ} \mathrm{C}$ ). Both HN functions were used with $b_{H N}=1$. When $b_{H N}=1$, eq 2 reduces to the ColeCole equation, ${ }^{37}$ which describes processes with symmetric distribution of relaxation times, characteristic of the secondary relaxations. 
The HN parameters of the three secondary relaxations were determined at several temperatures from a multiple non-linear regression analysis of the dielectric loss. The three characterizing peak parameters $\left(\Delta \varepsilon_{i}, \tau_{H N i}, a_{H N i}\right)$ were allowed to vary. As an example, Figure 6 plots the experimental data, the global fit and the deconvolution obtained in the fitting procedure of the loss factor at -45 and $-105^{\circ} \mathrm{C}$.
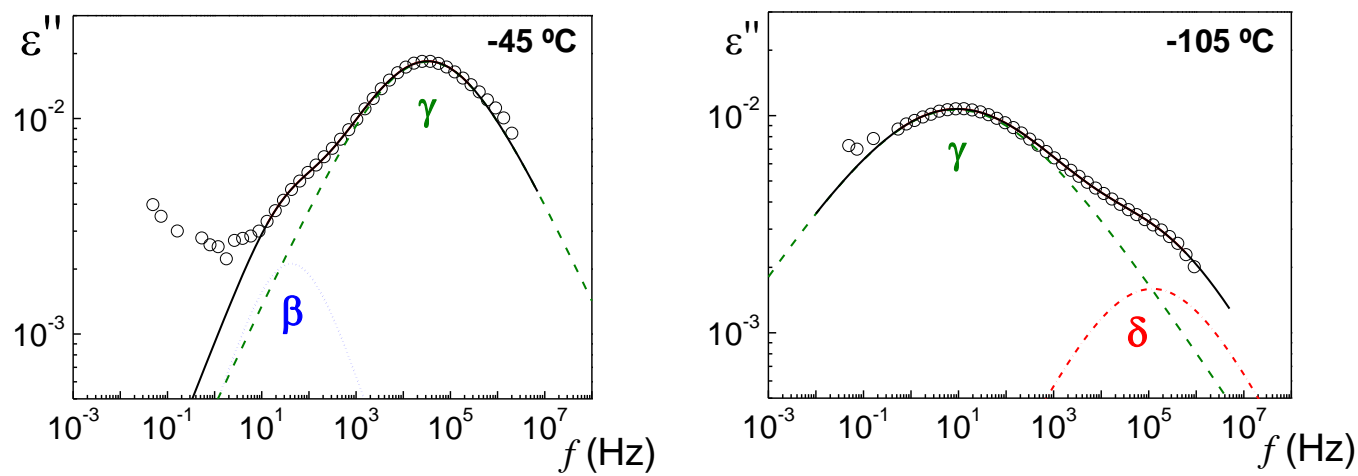

FIGURE 6 Experimental dielectric loss factor data (circle), global fit (continuous line) and individual relaxations (dashed lines) as a function of frequency at -45 and $-105^{\circ} \mathrm{C}$.

In the low frequency zone, where the conductive process is dominant, we have included a new term in order to characterize the spectrum: $\varepsilon^{*}(\omega)=\varepsilon^{*} \operatorname{dip}+\varepsilon^{*}$ cond with $\varepsilon^{*}$ cond $(\omega)=j\left(\sigma / \varepsilon_{0} \omega^{5}\right)$, where $\varepsilon_{0}$ $=8.854 \mathrm{pF} \mathrm{m}^{-1}$ is the dielectric permittivity of vacuum, $\sigma$ is the conductivity arising from charges transport at the liquid-electrode interface and $s$ is a constant $(s \leq 1)$. The dielectric analysis in this region was carried out by means of three $\mathrm{HN}$ functions and a conductive function, corresponding in decreasing order of frequency to $\gamma, \alpha$, MWS and EP processes. The fit parameters were determined at several temperatures from a multiple nonlinear regression analysis of the experimental data by using the following expression 


$$
\varepsilon^{\prime \prime}=\operatorname{Im}\left[\varepsilon_{\infty}+\sum_{i=1}^{3} \frac{\Delta \varepsilon_{i}}{\left(1+\left(i \omega \tau_{i}\right)^{\left.a_{H N i}\right)^{b_{H N i}}}\right.}+j\left(\frac{\sigma}{\varepsilon_{0} \omega^{s}}\right)\right]
$$

Figure 7 shows, as an example, the deconvolution of the experimental data at one temperature.

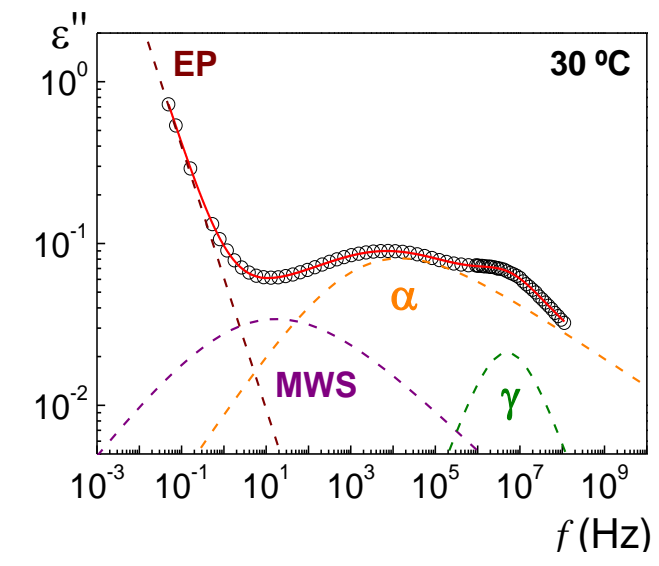

FIGURE 7 Experimental dielectric loss factor data (circle), global fit (continuous line) and individual relaxations (dashed lines) as a function of frequency at $30^{\circ} \mathrm{C}$.

The HN and ionic conductivity fit parameters obtained for all the processes observed in PUPH sample are plotted in Figures 8 to 10.

Figure 8 shows the $a_{H N}$ shape parameter of the PUPH relaxation processes. For the secondary relaxations, it is observed a slightly increase of the $a_{H N}$ values with the temperature. For the $\alpha$ relaxation, a low temperature dependence of the $a_{\mathrm{HN}}$ parameter value was observed, whereas the $b_{\mathrm{HN}}$ parameter increases with the temperature obtaining a value of 1 for the highest temperatures. In the case of the MWS process, the $a_{\mathrm{HN}}$ and $b_{\mathrm{HN}}$ parameters show also low temperature dependence. 
As we can observe, the secondary relaxation detected in the high temperature zone is well correlated with the $\gamma$ process previously characterized in the low temperature and high frequency range. For this process, $a_{H N}$ parameter increases with temperature.

At high temperatures and low frequencies the spectrum is very complicated, appearing closely overlapping dielectric relaxation processes ( $\alpha$ and $\gamma$ ) and conductive processes (MWS and EP). For this reason, although the shape parameters reported in the figure 8 are the obtained in our fitting procedure, it is possible that the sharp variations with temperature of some of them related to the complexity of the fitting procedure with a large number of variable parameters.

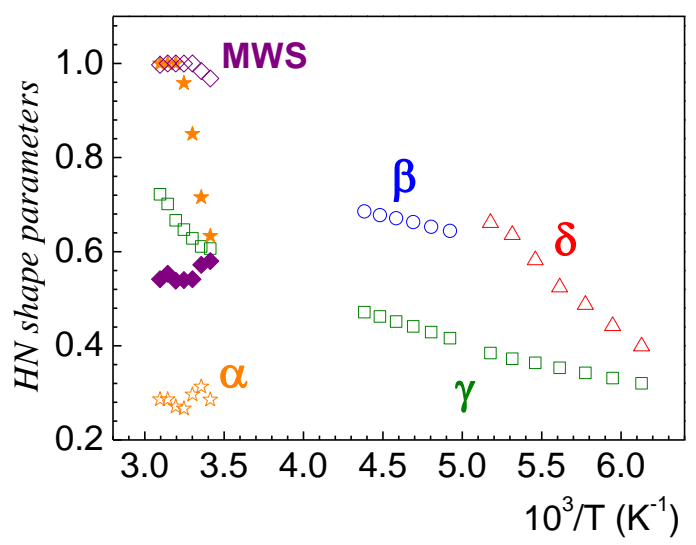

FIGURE 8 Temperature dependence of the shape parameters, $a_{H N}$ (open) and $b_{H N}$ (solid) from eq 2 , of the $\delta$ (triangles), $\gamma$ (square), $\beta$ (circles) and $\alpha$ (star) relaxations for PUPH.

Figure 9 shows the temperature dependence of the relaxation strength for the PUPH relaxation processes. Regarding the secondary relaxations, $\Delta \varepsilon$ of the $\gamma$ process slightly increases with temperature, following the classical trends. On the other hand, the strength values of the $\delta$ and $\beta$ processes are lower than that corresponding to the $\gamma$ process and remains practically constant with temperature. 
The $\alpha$-relaxation strength remains practically constant with temperature, following the classical trend. The thermal energy disturbs the alignment of the molecule dipoles that intervene in the cooperative motions and give rise to the relaxation. On the other hand, the strength of the MWS process also remains practically constant with temperature.

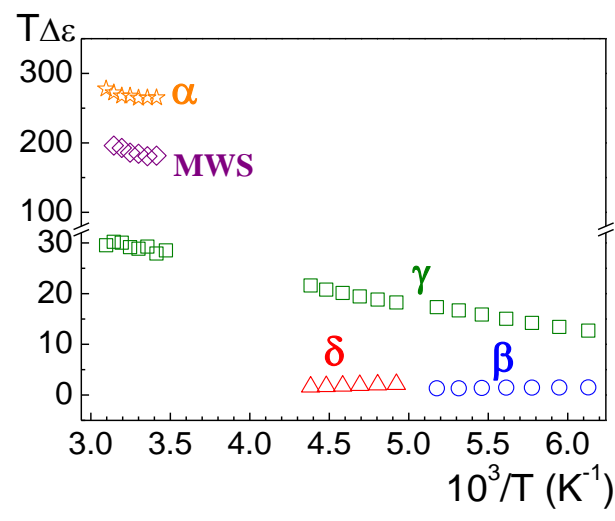

FIGURE 9 Temperature dependence of the strengths of the $\delta$ (triangles), $\gamma$ (square), $\beta$ (circles), $\alpha$ (star) and MWS (diamond) relaxations for PUPH.

Figure 10 shows the temperature dependence of the relaxation times associated with the peak maxima of the PUPH relaxation processes.

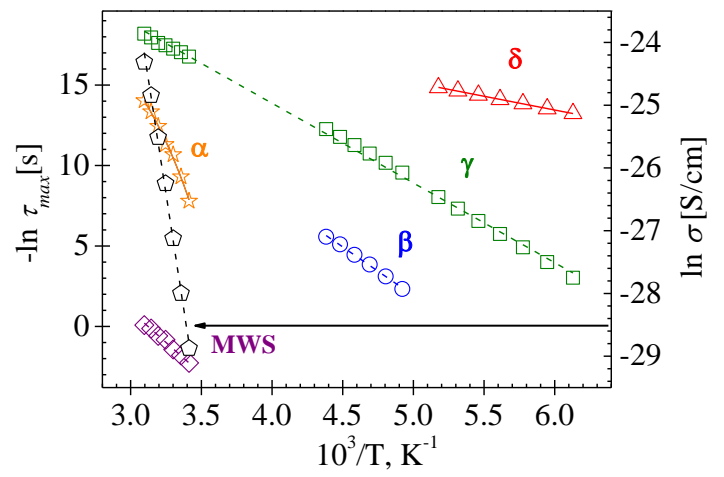

FIGURE 10 Arrhenius plot for the $\delta$ (triangles), $\gamma$ (square), $\beta$ (circles), $\alpha$ (star), MWS (diamond) and ionic conductivity (pentagon) processes for PUPH. 
The characteristic relaxation time $\tau_{\mathrm{HN}}$ is related to the relaxation times associated with the loss peak maxima, $\tau_{\max }, \mathrm{by}^{38}$

$$
\tau_{\max }=\tau_{H N}\left[\frac{\sin \left(\pi a_{H N} b_{H N}\left(2+2 b_{H N}\right)\right)}{\sin \left(\pi a_{H N}\left(2+2 b_{H N}\right)\right)}\right]^{1 / a_{H N}}
$$

It can be seen that the three secondary relaxations exhibit an Arrhenius (ARRH) behaviour: $\tau_{\text {max }}=\tau_{0} \cdot \exp \left[E_{a} / R T\right]$. The fit parameters to the ARRH equation are compiled in Table 1 . The activation energy values obtained for the $\gamma$ and $\delta$ processes $\left(41.2 \pm 0.4\right.$ and $14.2 \pm 0.1 \mathrm{~kJ} \mathrm{~mol}^{-1}$, respectively) are of the same order of magnitude than those obtained for other related polyurethanes. ${ }^{39}$ These processes are probably associated with the local motions of the main chain and/or the carbonate group in the soft phase. On the other hand, a similar weaker and slower $\beta$ process has been observed in a wide variety of water-contained systems. It has been associated with reorientational motions of the water molecules. ${ }^{40-42}$ This process has also been observed in other polyurethane systems ${ }^{39}$, although the samples had been extensively dried prior to the dielectric measurements, like in the case of our samples.

The temperature dependence of the relaxation time for the $\alpha$-process follows a VogelTammann-Fulcher-Hesse (VTFH) behaviour. ${ }^{43-49}$

$$
\tau=\tau_{0} \cdot \exp \left(\frac{D_{0} \cdot T_{v}}{T-T_{v}}\right)
$$

where $\tau_{0}$ is a pre-exponential factor identified sometimes with vibrational lifetimes ${ }^{50}$ (whose value is of the order of $\left.10^{-14} \mathrm{~s}\right), D_{0}$ is the strength coeficient, ${ }^{51,52}$ and $T_{v}$ is the Vogel temperature, currently associated with the temperature at which the entropies of the glassy system and the 
crystal are similar, i.e. when the configurational entropy of the glassy system is zero. The fit parameters of eq 5 to the Arrhenius plot (see Fig. 10) are summarized in Table 1. As usual, the results for $T_{v}$ are nearly $50 \mathrm{~K}$ below those of $T_{g}$. Moreover, the fragility parameter obtained for this polymer is lower than 10 , the frontier that separates fragile $\left(D_{0}<10\right)$ from strong $\left(D_{0}>10\right)$ glasses. ${ }^{51-55}$

TABLE 1 Characteristic parameters of the dipolar and conductivity processes for PUPH.

\begin{tabular}{ccccccc}
\hline & $\sigma_{\mathrm{dc}}$ & MWS & $\alpha$ & $\beta$ & $\gamma$ & $\delta$ \\
\hline $\log _{10} \tau_{0}, \mathrm{~s}$ & & $-10.6 \pm 0.1$ & $-11.9 \pm 0.2$ & $-14.0 \pm 0.3$ & $-14.6 \pm 0.1$ & $-10.3 \pm 0.1$ \\
$\mathrm{D}_{0}$ & - & $5.6 \pm 0.1$ & - & - & - \\
$\mathrm{T}, \mathrm{K}$ & & - & $228.0 \pm 0.2$ & - & - & - \\
$\mathrm{E}_{\mathrm{a}}, \mathbf{k J ~ m o l} \mathbf{~ m o l}^{-1}$ & $121.6 \pm 3.1$ & $64.9 \pm 6.0$ & - & $50.3 \pm 1.1$ & $41.2 \pm 0.4$ & $14.2 \pm 0.1$ \\
$\phi_{\mathrm{g}} / \mathrm{B}$ & & - & $0.039 \pm 0.001$ & - & - & - \\
$\alpha \cdot 10^{4}, \mathrm{~K}^{-1}$ & & - & $7.87 \pm 0.21$ & - & - & - \\
$\log _{10} \sigma_{0}, \mathbf{S ~ c m}^{-1}$ & $-11.18 \pm 0.5$ & & & & & \\
\hline
\end{tabular}

By comparing eq 5 with the Doolittle expression, ${ }^{56-57}$ the fraction of free volume at the glass transition temperature, $\phi_{g} / B$, and the free volume expansion coefficient $\alpha_{f}=(1 / V)(\partial V / \partial T)_{p}$ are estimated from the following expressions

$$
\begin{aligned}
& \frac{\phi_{g}}{B}=\frac{T_{g}-T_{v}}{D_{0} \cdot T_{v}} \\
& \frac{\alpha_{f}}{B}=\frac{1}{D_{0} \cdot T_{v}}
\end{aligned}
$$

According to the Cohen-Turbull theory, $B$ is a parameter close to unity related to the ratio between the critical volume for a relaxation process to take place and the volume of the segments involved in the process. ${ }^{58-59}$ Assuming $B=1$, the value of the relative free volume $\phi_{g}$ 
at $T_{g}$ is $0.039 \pm 0.001$, whereas the value of the thermal expansion coefficient of the free volume $\alpha_{f}$ at $T_{g}$ is $(7.87 \pm 0.21) \cdot 10^{-4} \mathrm{~K}^{-1}$. It is worth noting that the value of $\phi_{g}$ is slightly higher than the value reported for this quantity for most flexible polymers. Presumably, this is due to the fact that the relaxation curves only extend over a rather limited span of frequency and temperature windows. On the other hand, the thermal expansion coefficient of the free volume $\alpha_{f}$ at $T_{g}$ is similar to the value reported for the most flexible polymers, ${ }^{60}$ which lies in the vicinity of $5 \cdot 10^{-4}$ $\mathrm{K}^{-1}$.

The temperature dependence of the relaxation time for MWS process was ARRH-type. The characterized parameters obtained from the fitting of MWS relaxation are summarized in Table 1.

In our analysis, the $s$ parameter of eq 3 was equal to one for all analysed isotherms. Moreover, the conductivity values $(\sigma)$ obtained at several temperatures show an Arrhenius behaviour $\sigma_{d c}=\sigma_{0} \cdot \exp \left[-E_{a} / R T\right]$ (see Fig. 10), from which we have evaluated the activation energy obtaining a value of $121.6 \pm 3.1 \mathrm{~kJ} \mathrm{~mol}^{-1}$.

In order to analyse the conductive processes, it is interesting to investigate the dependence on frequency and temperature of the ac conductivity $\sigma^{\prime}$ ac $\left(=2 \cdot \pi \cdot f \cdot e_{0} \cdot \varepsilon^{\prime \prime}\right)$. Figure 11 shows the frequency plots of $\sigma_{a c}^{\prime}$ at temperatures between 30 to $140{ }^{\circ} \mathrm{C}$ (step $10{ }^{\circ} \mathrm{C}$ ) and the frequency dependence of $\sigma^{\prime \prime}$ at $140^{\circ} \mathrm{C}$. 


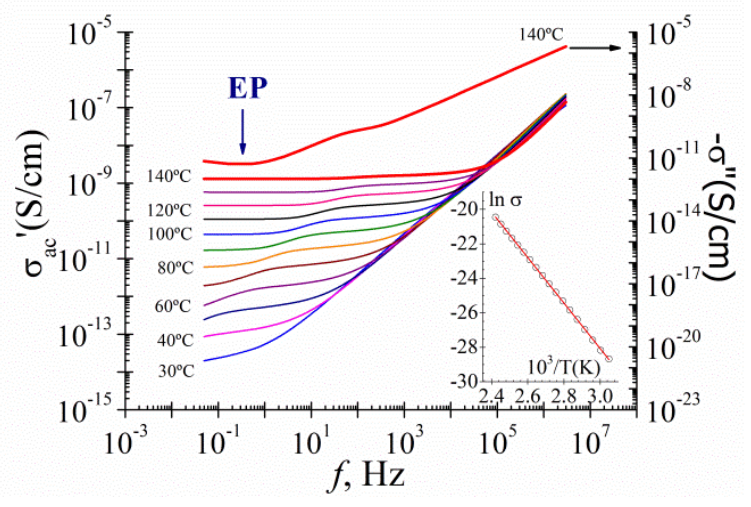

FIGURE 11 Values of ac conductivity against frequency for PUPH at temperatures in the range of $30^{\circ} \mathrm{C}$ to $140{ }^{\circ} \mathrm{C}$. Inset: Arrhenius plot associated with the dc conductivity.

As we can see, $\sigma_{a c}^{\prime}$ changes drastically with temperature and frequency. At temperatures higher than $70{ }^{\circ} \mathrm{C}$, a plateau (a region where $\sigma^{\prime}$ ac is frequency independent) can be clearly visualized in the plots. This behaviour is the same as the respective one of 'pure' dc conductivity. At higher frequencies, $\sigma_{a c}^{\prime}$ increase rapidly with increasing frequency. The transition region from dc (frequency independent) to ac (frequency dependent) conductivity shifts to higher frequencies with increasing temperature. As the temperature increases, the plateau is shifted to higher $\sigma_{a c}^{\prime}$ values, as expected for thermal activated conductivity.

Moreover, at high temperatures and low frequencies, $\sigma^{\prime \prime}$ decreases with decreasing frequency passing through a minimum and increasing again at the lowest frequencies. This behaviour can be ascribed to electrode polarization (EP). As the frequency decreases, more and more charge carriers accumulate at the interface between the sample and the electrodes, which leads to the drop in ac conductivity at low frequencies. The EP effect becomes increasingly important at high temperatures, reflecting the enhancement of the mobility of charge carriers. 
The temperature dependence of the dc conductivity values at several temperatures is described by the ARRH equation. These dc conductivity values are obtained from extrapolation to low frequencies of the frequency dependence of $\sigma^{\prime}$. The corresponding Arrhenius plot is shown in the inset of Figure 11, and their corresponding activation energy and $\log _{10} \sigma_{o} \mathrm{~S} \mathrm{~cm}-1$ are $118.5 \pm 1.0 \mathrm{~kJ} \mathrm{~mol}^{-1}$ and $11.1 \pm 0.2$, respectively. These values are in accordance with those obtained by fitting of the experimental data to eq 3 .

\section{CONCLUSIONS}

DSC results indicate a great degree of miscibility between the soft and hard segment phases due to the observed increase in the $T_{g}$ value of the PUPH with respect to the soft segment one, $\mathrm{PH} 100$. The endotherm transition at $160{ }^{\circ} \mathrm{C}$ indicates a long-range ordering of the hard domains.

The relaxational behavior of the PUPH has been analyzed by using Broad-Band Dielectric Spectroscopy. The spectrum exhibits five relaxations, in the range of frequencies and temperatures studied, named in increasing order of frequency MWS, $\alpha, \beta, \gamma$ and $\delta$.

At high temperatures and low frequencies the loss permittivity spectrum is dominated by EP, a conductivity process related to the accumulation of charges at the electrodes-polymer interface. The contribution to the dielectric loss in the EP process scales as ws. The spectrum expressed in terms of the tan $\delta$ exhibits a process presumably caused by the motion of macrodipoles arising from the concentration of charges in the electrode-liquid interfaces.

The MWS process is related to the build-up of charges at the interfaces of soft and hard microdomains components of the PUPH heterogeneous system. This process is not observed in 
the relaxational mechanical spectra. The absence of the MWS process in the mechanical relaxations spectrum is indicative of the conductive origin of this process. Evidence of the presence of these microdomains in PUPH under study has been demonstrated by means of the WAXS measurement results. ${ }^{12}$

On the other hand, at low temperatures and high frequencies, four dipolar relaxations are observed. For the $\alpha$ relaxation, the $T_{g}$ value obtained by MDSC and DRS (at $1 \mathrm{~Hz}$ ) techniques are in agreement. The $\beta$ process has been associated with reorientational motions of the water molecules. On the other hand, the $\gamma$ and $\delta$ processes are associated with local motions of the main chain and/or the carbonate group in the soft phase.

\section{ACKNOWLEDGEMENTS}

This work was financially supported by the DGCYT through Grant MAT2012-33483. The authors thank UBE Chem. Corporation for supplying the polycarbonatodiol to synthetize the polyurethanes of this work.

\section{REFERENCES AND NOTES}

1. Lee, S. in Thermoplastic Polyurethane Markets in the EU-Production, Technology, Applications and Trends a report from Rapra's Industry Analysis and Publishing Group, 1998.

2. Szycher, M. in Handbook of polyurethanes, Ed. CRC Press: Washington DC, 1999.

3. Oertel, G. in Polyurethane Handbook, Ed. Hanser $2^{\text {nd }}$ edition: New York, 1993.

4. Prisacariu C, Polyurethane Elastomers. From morphology to mechanical aspects, Springer, New York, 2011. 
5. Król, P. Progress in Materials Science 2007, 52, 915-1015.

6. Strawhecker, K. E.; Hsieh, A.J.; Chantawansri, T.L.; Kalcioglu, Z.I.; Van Vliet, K.J. Polymer 2013, 54(2), 901-908.

7. Costa, V.; Nohales, A.; Félix, P.; Guillem, C.; Gómez, C.M. Journal of Elastomers \& Plastics 2012, 45(3), 217-238.

8. Eceiza, A.; Martin, M.D.; de la Caba, K.; Kortaberria, G.; Gabilondo, N.; Corcuera, M.A.; Mondragon, I. Polym. Eng. Sci. 2008, 48, 297-306.

9. Kojio, K.; Nonaka, Y.; Masubuchi, T.; Furukawa, M. J. Polym. Sci: Part B: Polym. Phys. 2004, $42,4448-4450$.

10. Tanaka, H.; Kunimura, M. Polym. Eng. Sci. 2002, 42, 1333-1349.

11. Costa, V.; Muñoz, B.; Nohales, A.; Félix, P.; Gómez, C.M.; Guillem, C.; Latorre, J. Revista de Plásticos Modernos, Mayo 2008, 95(623), 384-389.

12. Gómez, C.M.; Culebras, M.; Cantarero, A.; Redondo-Foj, B.; Ortiz-Serna, P.; Carsí, M.; Sanchis, M.J. Applied Surface Science 2013, 275, 295- 302.

13. Pissis, P.; Georgoussis, G.; Bershtein, V.A.; Neagu, E.; Fainleib, A.M. J. Non-Cryst. Solids 2002, $305,150-158$.

14. P. Czech, L. Okrasa, F. Mechin, G. Boiteux, J. Ulanski, Polymer 2006, 47, 7207-7215.

15. Okrasa, L.; Czech, P.; Boiteux, G.; Mechin, F.; Ulanski, J. Polymer 2008, 49, 2662-2668.

16. Polizos, G.; Kyritsis, A.; Pissis, P.; Shilov, V.V.; Shevchenko, V.V. Solid State Ionics 2000, 136$137,1139-1146$.

17. Castagna, A.M.; Fragiadakis, D.; Lee, H.; Choi, T.; Runt, J. Macromolecules 2011, 44, 78317836. 
18. Castagna, A.M.; Pangon, A.; Choi, T.; Dillon, G.P.; Runt, J. Macromolecules 2012, 45, 84388444.

19. Fragiadakis, D.; Runt, J. Macromolecules 2013, 46, 4184-4190.

20. D’Arlas, B.F.; Rueda, L.; De la Caba, K.; Mondragon, I.; Eceiza, A. Polymer Engineering and Science 2008, 48 (3), 519-529.

21. Lazic, N.L.; Budinski-Simendic, J.; Ostojic, S.; Kicanovic, M.; Kicanovic, M.B.; Plavsic, M.B. Mater Sci Forum 2007, 555, 473-478.

22. Raftopoulos, K.N.; Janowski, B.; Apekis, L.; Pielichowski, K.; Pissis, P. European Polymer Journal 2011, 47, 2120-2133.

23. Pavlicevic, J.; Spirkova, M.; Strachota, A.; Mészáros Szécsényi, K.; Lazi'c, N.; BudinskiSimendi'c, J. Thermochimica Acta 2001, 509, 73-80.

24. Wang, L.F. European Polymer Journal 2005, 41, 293-301.

25. Rogulska, M.; Kultys, A.; Podkos'cielny, W. European Polymer Journal 2007, 43, 1402-1414

26. Jin-Chao, Z.; Fei-Peng, D.; Xing-Ping, Z.; Wei, C.; Xiao-Mei, W.; Hong, Z.; Xiao-Lin, X.; YiuWing, M. Composites: Part B 2011, 42, 2111-2116.

27. Maxwell, J.C. in Electricity and Magnetism, Clarendon, Oxford, 1893.

28. Wagner, K.W. Arch. Elektrotech. 1914, 2, 371-387.

29. Sillars, R.W. Inst. Electr. Eng. 1937, 80, 378-394.

30. Satti, G.; McLachlan, D.S. J. Mater. Sci. 2007, 42, 6477-6488.

31. Hodge, I.M.; Ngai, K.L.; Moynihan, C.T. Journal of Non-Crystalline Solids 2005, 351(2), 104115.

32. Pathmanathan, K.; Johari, G.P. J. Chem. Phys 1991, 95(8), 5990-5998. 
33. Havriliak, S.; Negami, S. Polymer 1967, 8(4), 161-210.

34. Havriliak, S.; Negami, S. J. Polym. Sci. Polym. Symp. 1966, 14, 99-117.

35. Havriliak, S.; Havriliak, S.J. in Dielectric and Mechanical Relaxation in Materials, Hanser: Munich, 1997, p 57.

36. Donth, E. J. Polym. Sci. Polym. Phys. 1996, 34(17), 2881-2892.

37. Cole, K.S.; Cole, R.H. J. Chem. Phys. 1941, 9, 341-352.

38. Boersma, A.; van Turnhout, J.; Wübbenhorst, M. Macromolecules 1998, 31, 7453-7460.

39. Fragiadakis, D.; Runt, J. Macromolecules 2013, 46, 4184-4190.

40. Cerveny, S.; Alegría, A.; Colmenero, J. Phys Rev. 2008, E 77, 031803-031807.

41. Capaccioli, S.; Ngai, K.L.; Shinyashiki, N. J. Phys. Chem. B 2007, 111, 8197-8209.

42. Fragiadakis, D.; Runt, J. Macromolecules, 2010, 43, 1028-1034.

43. Vogel, H. Z Phys. 1921, 22, 645-646.

44. Fulcher, G.S. J Am Ceram Soc. 1925, 8, 339-340.

45. Tamman, G.; Hesse, W. Z Anorg Allg Chem. 1926, 156, 245-247.

46. Lunkenheimer, P.; Kastner, S.; Köhler, M.; Loidl, A. Phys. Rev. E 2010, 81, 051504.

47. Martinez-Garcia, J. C.; Rzoska, S. J.; Drozd-Rzoska, A.; Martinez-Garcia J. Nat. Commun. 2013, 4, 1823.

48. Bauer, Th.; Lunkenheimer, P.; Loidl, A. Physical Review Letters 2013, 111, 225702.

49. Chakrabarty, S.; Karmakar, S.; Dasgupta, C. Annu. Rev. Condens. Matter Phys. 2014, arXiv:1404.2701 [cond-mat.stat-mech].

50. Lunkenheimer, P.; Schneider, U.; Brand, R.; Loidl, A. Contemp. Phys. 2000, 41: 15-36.

51. Böhmer, R. ; Ngai, K. L.; Angell, C. A.; Plazek, J. D. J. Chem. Phys. 1993, 99, 4201-4209. 
52. Angell, C.A. Science 1995, 267, 1924-1935.

53. Angell, C.A. Polymer 1997, 38, 6261-6266.

54. Angell, C.A. in Complex Behavior of Glassy Systems, Proceedings of the XIV Sitges Conference, Sitges, Barcelona, Spain, 1996.

55. Rubi, M. ; Pérez-Vicente C., Lecture Notes in Physics Vol. 492. Complex Behaviour of Glassy Systems. Proceedings, Barcelona, Spain, Eds.Springer, Berlin, 1997.

56. Doolittle, A. K. J. Appl. Phys. 1951, 22(12), 1471-1975.

57. Doolittle, A.K. J. Appl. Phys. 1952, 23(2), 236-423.

58. Bueche, F. J. Chem. Phys. 1959, 30, 748-752.

59. Cohen, M.H.; Turnbull D. J. Chem. Phys. 1959, 31, 1164-1169.

60. Ferry, J.D. Viscoelastic Properties of polymers, 2nd ed. John Wiley\&Sons: New York, 1961. 
P. Ortiz-Serna, M. Carsí, B. Redondo-Foj, M. J. Sanchis, M. Culebras, C.M. Gómez and A. Cantarero

\section{Thermal and Dielectric Properties of Polycarbonatediol Polyurethane}

The molecular mobility of a segmented polyurethane has been studied using Broad-Band Dielectric Spectroscopy (BBDS) and Modulated Differential Scanning Calorimetry (MDSC). The microphase-separated morphology associated with soft and hard domains is reflected in the dielectric spectra, at high temperatures, by the presence of the Maxwell-Wagner-Sillars (MWS) interfacial polarization process.

GRAPHICAL ABSTRACT FIGURE

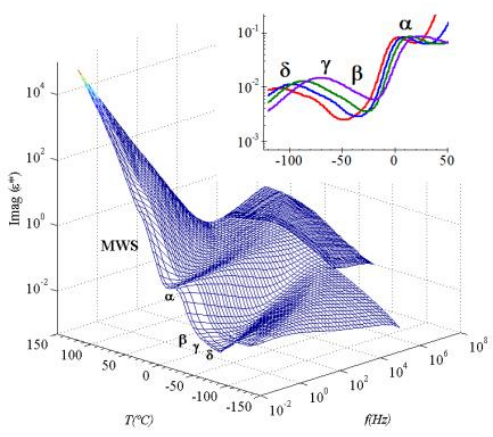

